\title{
Effects and mechanism of Tripterygium wilfordii on chronic glomerulo nephritis
}

\author{
W.Y. Pei ${ }^{1}$, C.H. Yang ${ }^{2}$ and X.L. Zhang ${ }^{3}$ \\ 'Department of Nephrology, Xinxiang Central Hospital, Xinxiang, China \\ ${ }^{2}$ MRI Room, The Third Affiliated Hospital of Xinxiang Medical University, Xinxiang, \\ China \\ ${ }^{3}$ Department of Nephropathy and Rheumatology, \\ The Third Affiliated Hospital of Xinxiang Medical University, Xinxiang, China \\ Corresponding author: W.Y. Pei \\ E-mail: xiaolizhangcn@163.com \\ Genet. Mol. Res. 15 (1): gmr. 15017410 \\ Received August 11, 2015 \\ Accepted November 13, 2015 \\ Published February 5, 2016 \\ DOI http://dx.doi.org/10.4238/gmr.15017410
}

ABSTRACT. The objective of this study was to investigate the clinical effects of Tripterygium wilfordii on chronic glomerulo nephritis (CGN) and its mechanisms. Eighty-two cases of CGN treated in our hospital were randomly divided into observation and control groups. The control group was treated with conventional western medicine, and the observation group was treated with conventional western medicine and orally-administered $T$. wilfordii pills for three courses of treatment, each consisting of 4 weeks. Changes in serum creatinine, blood urea nitrogen, blood total cholesterol, blood albumin, and 24-h urine protein were observed. The levels of peripheral tumor necrosis factor- $\alpha$ (TNF- $\alpha$ ) and interleukin-6 (IL-6) were determined with enzyme-linked immunosorbent assay. The curative effects of both groups were evaluated respectively. Both groups had significantly improved serum creatinine, blood urea nitrogen, blood total cholesterol, blood albumin, and 24-h urine protein $(P<0.05)$, and the observation group exhibited a more significant improvement $(P<0.05)$. TNF- $\alpha$ and IL-6 levels in both groups obviously decreased $(P<0.05)$, and the observation group exhibited remarkable changes $(P<0.05)$. After treatment, the total 
efficiency of the observation group was $90.24 \%$, which was significantly higher than the $73.17 \%$ of the control group $(P<0.05)$. In conclusion, $T$. wilfordii can significantly improve kidney function and clinical symptoms in CGN patients, and the mechanism is possibly related to its inhibition of the secretion of TNF- $\alpha$ and IL-6.

Key words: Tripterygium wilfordii; Chronic glomerulo nephritis; Curative effect

\section{INTRODUCTION}

CGN is also called chronic nephritis and can be described as albuminuria, hematuresis, hypertension, and edema with a slowly developing decline in renal function (Kasap et al., 2014). The development of CGN varies, and the disease is refractory with slow and hidden onset. Chronic sustained progress and recurrence may cause a secondary lesion of the renal tubular and interstitial, which progressively develops into CGN (Ding et al., 2013; Shi et al., 2013). Currently, CGN is the primary cause of end stage renal disease in China, but the causes remain unknown. According to clinical and experimental data, the pathogenesis of CGN is closely correlated with immunological mechanisms and immune function disorder is a contributing factor. Immune-mediated damage is the initiating factor in the pathogenesis of CGN, resulting in inflammation which is the major cause of CGN (Mostafidi and Ardalan, 2015; Roh et al., 2015). No satisfactory treatment methods for CGN have been found using western medicine. Immunosuppressive agents are largely used to prevent or delay the progressive aggravation of kidney function so as to relieve clinical symptoms and prevent severe complications. Therefore, the treatment of CGN usually focuses on comprehensive prevention and symptom treatment. Tripterygium wilfordii is a plant of the family Celastraceae. It has been shown that Tripterygium wilfordii has pharmacological properties such as anti-inflammatory, immunity regulation, anti-tumor, and reproduction suppression effects, so it is widely applied for treating rheumatoid arthritis, glomerular nephritis, lupus erythematosus, and many autoimmune and skin diseases with significant effects (Wang et al., 2015; Su et al., 2015). In this study, the clinical effects of $T$. wilfordii on CGN were observed, aiming to further investigate its mechanism of action.

\section{SUBJECTS AND METHODS}

\section{General data}

Eighty-two cases of CGN treated in our hospital from June 2012 to June 2014 were selected. All patients conformed to the diagnosis criteria of CGN. The exclusion criteria were: 1) creatinine $>177 \mu \mathrm{M}$ or endogenous creatinine clearance rate $<60 \mathrm{~mL} / \mathrm{min}$; 2) patients who were pregnant, nursing, or had excessive menstruation; 3) patients with coexisting reversible but untreated diseases; 4) patients with severe heart, lung, liver, and kidney incompetence; 5) patients with severe endocrine and hematopoietic system diseases; and 6) patients with psychosis. The patients were randomly divided into control and observation groups. The control group consisted of 41 cases, including 24 males and 17 females, ages 20-65 with an average age of $38.34 \pm 13.16$. The disease course was $1.5-15$ years, with a mean of $4.72 \pm 2.98$ years. There were 29 cases with edema, 25 cases with hematuria, 22 cases with hypertension, and 7 cases with incomplete kidney 
function. The observation group consisted of 41 cases, including 25 males and 16 females, ages 22-26 with an average age of $39.21 \pm 12.78$. The disease courses were 1.8-15.2 years with an average course of $4.81 \pm 3.02$ years. There were 27 cases with edema, 26 cases with hematuria, 20 with hypertension, and 8 with incomplete kidney function. The differences in age, gender, disease development, and complications between both groups were not statistically significant. This study was conducted in accordance with the Declaration of Helsinki. This study was conducted with approval from the Ethics Committee of the Third Affiliated Hospital of Xinxiang Medical University. Written informed consent was obtained from all participants.

\section{Treatment methods}

The control group was administered a valsartan capsule (Livzon group, Zhuhai, China) (Domestic Drugs Database No.: H20000622, Specification: $80 \mathrm{mg}$ ) orally-taken and $80 \mathrm{mg} / \mathrm{day}$ captopril tablets (Squibb Pharmaceuticals Company, Shanghai, China) (Domestic Drugs Database No.: H31022816, specification: $25 \mathrm{mg}$ ) orally-taken, $25 \mathrm{mg} / \mathrm{day}$. The blood pressure of the patients was strictly controlled. If the blood pressure was beyond the criteria, a $\beta$-receptor blocker or calcium antagonist was added to the regimen. Infections were actively controlled and prevented, and patients with a fever or a high score on routine blood tests were administered antibiotics. If there was a urinary tract infection, a middle urine culture was conducted, and antibiotics were selected according to drug sensitivity results. Patients with hyperlipemia were administered simvastatin. Disordered water electrolyte acid-base balance was addressed and taking low salt, low fat, and high quality protein foods was recommended. Treatment groups: on the basis of the conventional treatment of the control group, T. wilfordii tablets $(20 \mathrm{mg})$ were taken orally 3 times/day for 4 week courses, and there were 3 courses.

\section{Detection of serum creatinine, urea nitrogen, total cholesterol, albumin, and 24-h urine protein}

Before and after treatment, the fasting peripheral blood and 24-h urine of both groups were collected to detect serum creatinine (Scr), blood urea nitrogen (BUN), blood total cholesterol (TC), and blood albumin (ALB) with an automatic biochemistry analyzer (Hitachi Ltd, Tokyo, Japan). The 24-h urine protein (24-h uTP) was detected using the biuret method.

\section{Detection of peripheral tumor necrosis factor- $\alpha$ (TNF- $\alpha)$ and IL-6 protein levels}

Before and after treatment, the fasting peripheral blood of both groups was collected and subjected to $10 \mathrm{~min}$ of centrifugation at $4000 \mathrm{rpm}$. The supernatant was used for ELISA detection of TNF- $\alpha$ and IL-6 protein levels, according to the manufacturer protocol (Dingguo, Beijing, China). Three measurements were made for each sample and standard, and OD values were measured at $492 \mathrm{~nm}$ using a microplate reader.

\section{Curative effect evaluation}

The evaluation standards of curative effects were: cured: no clinical symptoms, normal 24-h UTP and kidney function; markedly effective: almost no clinical symptoms, decrease in 24-h UTP $\geq 40 \%$, almost normal kidney function (difference from the normal value $<15 \%$ ); effective: 
improved clinical symptoms, decrease in 24-h uTP < 40\%, improved kidney function; no effect: no improvement or aggravation of clinical syndromes, signs, 24-h uTP, and kidney function. The total efficiency $=($ cured + markedly effective + effective $) /$ total cases $\times 100 \%$.

\section{Statistical analysis}

All data were analyzed with the SPSS13.0 software (SPSS Inc., Chicago, IL, USA). The measured data are reported as means \pm SD and were compared with a Student $t$-test. The comparison of technology was conducted with a chi-square test. $\mathrm{P}<0.05$ indicates the difference was statistically significant.

\section{RESULTS}

\section{Comparison of biochemical indicators in both groups before and after treatment}

As shown in Figure 1, before treatment, the differences in indicators of the control group and the observation group are of no statistical significance $(P>0.05)$. After treatment, the levels of Scr, BUN, TC and $24 \mathrm{~h}$ uTP significantly decreased, while ALB level remarkably increased $(\mathrm{P}<$ $0.05)$, and the observation group had more significant improvement $(P<0.05)$.
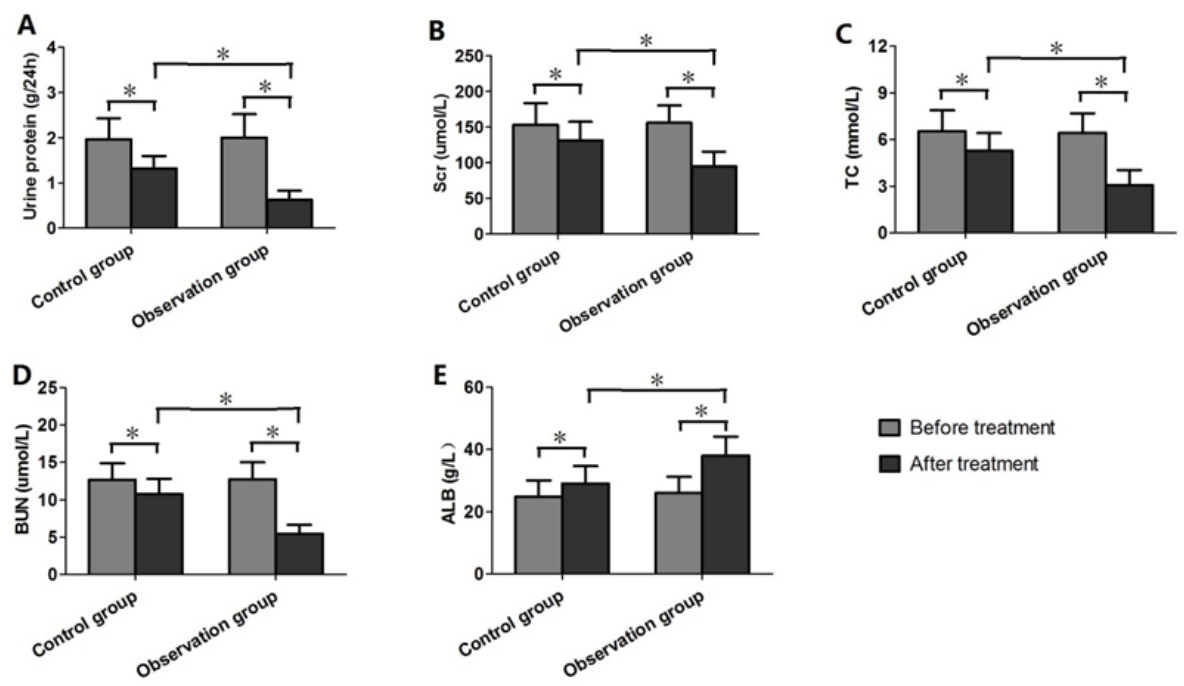

Figure 1. Comparison of biochemical indicators in both groups. A. Urine protein; B. Scr; C. TC; D. BUN; E. ALB.

\section{Comparison of peripheral TNF- $\alpha$ and IL- 6 protein levels in both groups before and after treatment}

Before treatment, the differences in peripheral TNF- $\alpha$ and IL-6 protein levels between both groups were not statistically significant $(P>0.05)$. After treatment, both groups had obviously decreased levels $(P<0.05)$, and the observation group had a more significant decrease $(P<0.05$; Figure 2). 
A

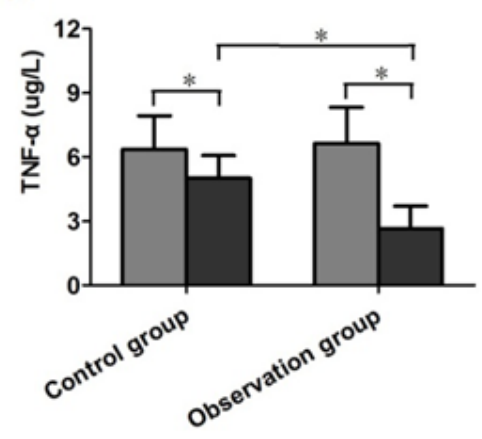

B

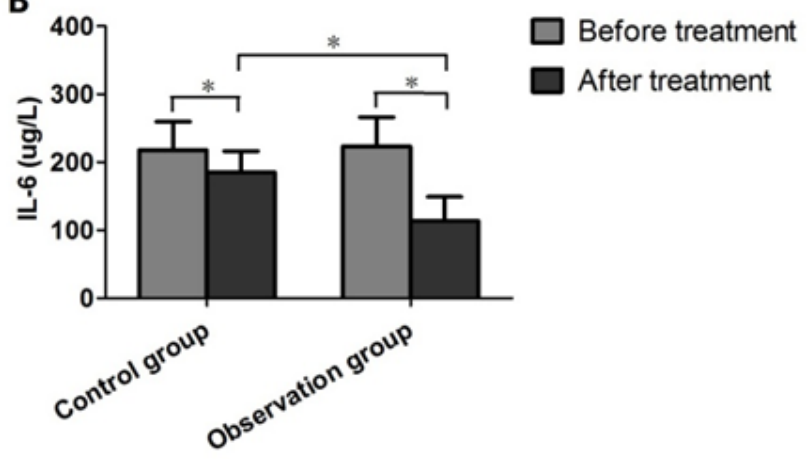

Figure 2. Comparison of peripheral blood TNF- $\alpha$ and IL-6 levels in both groups. A. TNF- $\alpha$; B. IL-6.

\section{Comparison of curative effects in both groups}

After treatment, the total efficiency of the observation group was $90.24 \%$, while that of the control group was $73.17 \%$. The observation group had a significantly greater total clinical efficiency than the control group $(\mathrm{P}<0.05$; Table 1$)$.

Table 1. Comparison of curative effects in two groups.

\begin{tabular}{l|c|c|c|c|c|c}
\hline Group & Cases & Cured & $\begin{array}{c}\text { Markedly } \\
\text { effective }\end{array}$ & Effective & No effect & $\begin{array}{c}\text { Total efficiency } \\
(\%)\end{array}$ \\
\hline Control group & 41 & 3 & 14 & 13 & 11 & 73.17 \\
\hline $\begin{array}{l}\text { Observation } \\
\text { group }\end{array}$ & 41 & 7 & 19 & 11 & 4 & 90.24 \\
\hline
\end{tabular}

\section{DISCUSSION}

CGN has a long disease course, high recurrence of symptoms, slow progression, and is the major chronic kidney disease in China. In Chinese adults, the prevalence rate of chronic kidney disease is around 10\%, constituting a major health threat (Poterucha et al., 2014). CGN has symptoms with different pathologic mechanisms. The disease is mainly caused by the glomerular injury mediated by an immune reaction, including humoral immunity and cell-mediated immunity. In the pathology of CGN, humoral immunity and cell-mediated immunity mechanisms play an important role, as the immune complex causes changes to GN (Sancho et al., 2010). As indicated by modern medicine research, CGN is related to disordered immune stability. When glomerular injury is caused, inherent cells are infiltrated, which activates some inflammatory mediators. The inflow of circulating white blood cells, synthesis of cytokines, and release of protein lytic enzymes cause the activation of coagulation interlocking reactions and the production of pro-inflammatory mediators. This results in bad kidney filtration function, affecting the discharge of toxic substances, increasing Scr and BUN in vivo, which aggravates the kidney disease (Mehta et al., 2013; Ye et al., 2014). 
TNF- $\alpha$ is generated by activated mononuclear cells and macrophages. As shown by research data, the expression of TNF- $\alpha$ in CGN increases, which stimulates glomerular cells, neutrophils, and vascular endothelial cells to synthesize inflammatory mediators. This gives a rise to glomerular capillary permeability, aggravates mononuclear cell and neutrophils infiltration, promotes the synthesis and secretion of TNF- $\alpha$, and leads to the interaction of the cytokine network (Santoro et al., 2012). IL-6 is an important inflammatory cytokine, functioning in the immune pathogenesis of CGN and accelerating glomerular sclerosis. The combination of IL-6 and the glomerular cell membrane receptor aggravates glomerular injury and cell apoptosis (Wu et al., 2010; Shen et al., 2012). Liu et al. (2014) observed the changes in inflammation in vivo by establishing the CGN model of aged rats. The results showed that the TNF- $\alpha$ and IL-6 expression of glomerular mesentery in the model group significantly increased. In this research, the peripheral TNF- $\alpha$ and IL- 6 protein levels of 82 CGN patients were higher than the normal value, which further demonstrates the roles of TNF- $\alpha$ and IL-6 in the occurrence and development of CGN, also indicating a possible synergistic reaction between TNF- $\alpha$ and IL-6.

The treatment method of CGN using Western medicine is insufficient. Many clinical reports show that treatment combining traditional Chinese medicine (TCM) and western medicine leads to significant curative effects which are better than that of TCM or western medicine alone. T. wilfordii is a Chinese herbal extract which has anti-inflammation and immunologic suppression properties and is extensively applied to treat rheumatic disease, systemic lupus erythematosus, inflammatory bowel disease, nephritis, and many immune diseases (Wan et al., 2010; Wan et al., 2012; Chen et al., 2013). In this research, conventional treatment combined with orally-taken $T$. wilfordii tablets resulted in a distinct decrease of UTP and peripheral SCr, BUN, and TC with a significant increase in the ALB level, and the improvement was greater than that of conventional treatment. UTP, Scr, BUN, and TC are the primary clinical indicators of the degree of kidney function. The results demonstrate that $T$. wilfordii can improve the clinical symptoms of CGN patients. The TNF- $\alpha$ and IL-6 levels in CGN patients are also significantly down-regulated, which indicates that $T$. wilfordii can be used to regulate the balance of cell-mediated immunity and humoral immunity, so as to inhibit the expression of TNF- $\alpha$, IL-6, and other inflammatory cytokines, and thus inhibit inflammatory reactions, improving capillary permeability. The results of this study in addition to previously published research data indicate that a complex synergistic relationship exists between TNF- $\alpha$ and IL-6. In the cytokine networks of CGN inflammatory reactions, they promote the development of CGN conditions. In summary, T. $m$ wilfordii can be used to suppress the expression of inflammatory cytokines in CGN patients and significantly change clinical symptoms.

\section{REFERENCES}

Chen XY, Gu C, Ma M, Cong Q, et al. (2014). A mouse model of premature ovarian insufficiency induced by tripterygium glycoside via subcutaneous injection. Int. J. Clin. Exp. Pathol. 7: 144-151.

Ding SY, Zheng PD, He LQ, Hou WG, et al. (2013). [The research on xiaochalhu decoction improving the inflammation of chronic glomerulonephritis patients and relieving the proteinuria]. Zhongguo Zhong Xi Yi Jie He Za Zhi 33: 21-26.

Kasap B, Çarman KB and Yiş U (2014). A case of acute post-streptococcal glomerulonephritis that developed posterior reversible encephalopathy syndrome. Turk Pediatri Ars 49: 348-352.http://dx.doi.org/10.5152/tpa.2014.430

Liu S, Liu LZ, Tan ZH and Yang XF (2014). Effect of TWP on the efficacy and inflammatory markers of glomerulonephritis in aged rats. Chin. J. Immunol. 30: 627-629.

Mehta K, More V, Chitale A and Khubchandani S (2013). Atypical hemolytic uremic syndrome with membranoproliferative glomerulonephritis. Indian Pediatr. 50: 793-794.

Mostafidi E and Ardalan MR (2015). Re: prognostic factors in crescentic glomerulonephritis: a single-center experience. Iran. J. Kidney Dis. 9: 336-337. 
Poterucha TJ, Wetter DA, Grande JP, Gibson LE, et al. (2014). A retrospective comparison of skin and renal direct immunofluorescence findings in patients with glomerulonephritis in adult Henoch-Schönlein purpura. J. Cutan. Pathol. 41: 582-587.http://dx.doi.org/10.1111/cup.12295

Roh M, Sohn JH, Kim TY, Kim SJ, et al. (2015). Gastric Syphilis and Membranous Glomerulonephritis. Clin. Endosc. 48: 256259.http://dx.doi.org/10.5946/ce.2015.48.3.256

Sancho A, Pastor MC, Bayés B, Sánchez A, et al. (2010). Posttransplant inflammation associated with onset of chronic kidney disease. Transplant. Proc. 42: 2896-2898.http://dx.doi.org/10.1016/j.transproceed.2010.07.075

Santoro D, Postorino A, Costantino G, Savica V, et al. (2012). Anti-TNF- $\alpha$ therapy in membranous glomerulonephritis. Clin. Kidney J. 5: 487-488.http://dx.doi.org/10.1093/ckj/sfs105 PubMed

Shen L, Lu G, Dong N, Jiang L, et al. (2012). Von Willebrand factor, ADAMTS13 activity, TNF- $\alpha$ and their relationships in patients with chronic kidney disease. Exp. Ther. Med. 3: 530-534.

Shi WW, Yu XJ, Chen LX, Tan LY, et al. (2013). Clinical study on the treatment for chronic glomerulonephritis with Liuwei dihuang Decoction. J. Pract. Med. 29: 1352-1354.

Su MX, Zhou WD, Lan J, Di B, et al. (2015). Rapid and sensitive analysis of multiple bioactive constituents in tripterygium glycosides tablets using liquid chromatography coupled with time-of-flight mass spectrometry. J. Sep. Sci. 38: 804-812. http://dx.doi.org/10.1002/jssc.201400946

Wan Y, Sun W, Zhang H, Yan Q, et al. (2010). Multi-glycoside of Tripterygium wilfordii Hook f. ameliorates prolonged mesangial lesions in experimental progressive glomerulonephritis. Nephron, Exp. Nephrol. 114: e7-e14. http://dx.doi.org/10.1159/000245061

Wan YG, Zhao Q, Sun W, Zhang HL, et al. (2012). Contrasting dose-effects of multi-glycoside of Tripterygium wilfordii HOOK. f. on glomerular inflammation and hepatic damage in two types of anti-Thy1.1 glomerulonephritis. J. Pharmacol. Sci. 118: 433-446.http://dx.doi.org/10.1254/jphs.11157FP

Wang J, Miao M, Zhang Y, Liu R, et al. (2015). Quercetin ameliorates liver injury induced with Tripterygium glycosides by reducing oxidative stress and inflammation. Can. J. Physiol. Pharmacol. 93: 427-433. http://dx.doi.org/10.1139/cjpp-2015-0038

Wu X, Xu J and Luo X (2010). [Effects of Tripterygium hypoglaucum on serum IL-1, IL-6, and TNF-alpha in chronic nephritis rats]. Zhongguo Zhong Yao Za Zhi 35: 3354-3356.

Ye CL, He AD and Huang LJ (2014). Evaluation of the efficacy and safety of huoxue yishen decoction and western medicine in treatment of chronic glomerulonephritis. Chin. J. Bioch. Med. 3: 125-127. 